\title{
Surrogate decision making of chemotherapy consent: do we really provide informed consent of chemotherapy for patients?
}

\author{
Jung Hye Kwon ${ }^{1}$, Sun Kyung Baek², Bong-Seog Kim³ , Su-Jin Koh", Hee Kyung Ahn ${ }^{5}$ Joo Han Lim ${ }^{6}$, \\ Chiyeon $\mathrm{Lim}^{7}$, and Do Yeun $\mathrm{Kim}^{8}$
}

\begin{abstract}
${ }^{1}$ Department of Internal Medicine, Kangdong Sacred Heart Hospital, Hallym University College of Medicine, Seoul; ${ }^{2}$ Department of Internal Medicine, Kyung Hee University School of Medicine, Seoul; ${ }^{3}$ Department of Internal Medicine, Veterans Health Service Medical Center, Seoul; ' ${ }^{4}$ Department of Hematology and Oncology, Ulsan University Hospital, Ulsan; ${ }^{5}$ Department of Internal Medicine, Gachon University Gil Medical Center, Incheon; ${ }^{6}$ Department of Internal Medicine, Inha University Hospital, Incheon; ${ }^{7}$ Department of Biostatistics, Dongguk University School of Medicine, Seoul;

${ }^{8}$ Department of Internal Medicine, Dongguk University llsan Hospital, Goyang, Korea
\end{abstract}

Received: July 19, 2017

Revised : December 23, 2017

Accepted: January 6, 2018

\section{Correspondence to}

Do Yeun Kim, M.D.

Department of Internal Medicine, Dongguk University Ilsan Hospital, 27 Dongguk-ro, Ilsandong-gu, Goyang 10326, Korea

Tel: +82-31-961-7143

Fax: +82-31-961-7141

E-mail: smdkdy@dumc.or.kr
Background/Aims: Despite increased demand for cancer patient's to make their own decisions based on an adequate understanding of what is involved in chemotherapy, the primary signing agent and the reasons for surrogate signing have not been appropriately evaluated.

Methods: The ethics committee of the palliative medicine subgroup of the Korean Cancer Study Group designed this study and solid cancer patients to whom chemotherapy was offered, from seven institutions, were evaluated. The details relating to surrogate's signing of chemotherapy consent were evaluated. Then, we analyzed the factors associated with surrogate's signing according to patient's demographics and characteristics related to chemotherapy consent.

Results: Surrogate's signing was noted for $20.7 \%$ (84/405) of patient and over half of surrogate signings were performed by the patients' son or daughter (60.7\%). Two main reasons for surrogate signing were patient's incapacity (34.5\%) and taking over authorization from patients (33.3\%). The factors associated with more frequent surrogate's signing were absence of spouse, lower education level, outpatient, and when residents played a role as a principle provider of chemotherapy consent.

Conclusions: This study suggests the lack of patients' own decision making for chemotherapy in some situations. This ethical dilemma must be considered for adequately informed decision making for chemotherapy while ensuring the patients' autonomy is maintained.

Keywords: Informed consent; Surrogate decision making; Drug therapy; Neoplasms

\section{INTRODUCTION}

Informed consent is hospital proof of patients' autonomy and has a role with legal ramifications, also $[1,2]$. To make informed decisions, cancer patients need to have a reasonable expectation about the treatment costs and benefits based on information provided by their physician. This process runs continuously during their disease trajectory from initial diagnosis to cure or end of life care.

The contents of any informed consent consists several basic elements such as the patient's diagnosis, treatment 
goal, treatment benefit, treatment toxicity, alternatives to proposed treatment, and agreement of patients or their surrogates [3]. Nowadays, acquiring chemotherapy consent for cancer patients who are planning to have chemotherapy is standard practice. According to guideline of American Society of Clinical Oncology, having a chance to ask questions, and receiving a copy of this consent form are listed as essential elements of informed consent, also. Meanwhile, a principal person to sign the document is designated by the patient and a person reading/translating in the case of patients requiring translation or a verbal reading [4]. Recommended chemotherapy consent forms of the Korean academy of medical sciences suggest that patient do sign subsequent sentences like 'I have heard my diagnosis/treatment plan etc.' for each session after any explanation of diagnosis, treatment plan, alternative options, and adverse effects [5]. A signed copy is also supposed to be provided to the patient and/or their surrogates.

The person who takes medical information on behalf of the patient is extremely important when we consider medical law that states, 'physician should acquire autonomous patient consent after full explanation of disease, treatment plan, side effects, and all invasive treatments including the administration of medications' [6]. It means patients should be given opportunities to decide for themselves which treatments they wish to undergo in light of full understanding of information in advance. However, several studies observed that some fully conscious cancer patients tend to entrust their decision to their family member or their physician [7-9]. Patient's own decision making may be impossible in situations like a sudden loss of consciousness or rapidly deteriorating situation, also. A recent longitudinal study for Korean advanced cancer patients demonstrated that surrogate signed consent on behalf of patients was significantly frequent for do not resuscitate directives, emergency hemodialysis, and intensive care unit admission compared with chemotherapy, radiotherapy, and diagnostic test [10]. Authors suggest that health care professionals should consider the significant roles familial surrogate play in end of life issues. Because this study did not focus exclusively on issues for surrogate signing for chemotherapy consent, the proportion and the reasons concerning surrogate signing for chemotherapy consent are still unknown.
We frequently observed family members of patients requesting that physicians keep a cancer diagnosis 'secret' or to tell the patients their chemotherapy aim as 'curative' instead of life prolonging and or for symptom control with palliative aims. Though patient autonomy is now weighted more heavily, including the 'advance directive' in our society, the real situation often conflicts with meeting effective informed decision making.

This ethical dilemma is not easily solved and studies about these issues are lacking, even worse in Korea. Therefore, the ethics subcommittee of palliative medicine subgroup of Korean Cancer Study Group (KCSG) planned this analysis of current practice for obtaining chemotherapy consent.

This study aims to analyze the proportion of and factors associated with a surrogate's signing, upon acquisition of chemotherapy consent.

\section{METHODS}

\section{Design}

The seven medical oncologists of the ethics committee of the palliative medicine subgroup among the KCSG completed this study design after several rounds of feedback. We reviewed chemotherapy consents from cancer patients bearing solid tumors who had signed such consents during the period between July 2014 and December 2014 from institutions. This study was approved by the Institutional Review Board (Dongguk University Ilsan Hospital protocol number 2015-74) at each study center.

\section{Data collection}

Consecutive patients with various types of solid cancers, to whom chemotherapy was offered, were included. Patients' demographics including age, sex, presence of spouse, presence of a child, having a religion, education level, stage of the cancer, chemotherapy goals and type of cancer, were obtained.

We checked whether chemotherapy consent forms included diagnosis, treatment aim, treatment goal, adverse effects, other alternative options, principal agent for signing, and sign by information taker and provider. The place of acquisition and principal provider of chemotherapy consent were also recorded. We filled out the 
relationship of surrogate by replacing patient's signing and the reason replacing the patients' signing, if the surrogate is the principle receiver of chemotherapy informed consent.

\section{Statistical analysis}

Demographic and clinical characteristics were expressed by descriptive statistics for 405 subjects. Continuous variables were expressed using descriptive statistics (mean \pm $\mathrm{SD}$ ) and categorical variables were described by number

Table 1. Patient characteristics $(n=405)$

\begin{tabular}{|c|c|}
\hline Variable & Value \\
\hline Age, yr & $61.82 \pm 11.85$ \\
\hline Sex, female/male & $161 / 244(39.8 / 60.2)$ \\
\hline Presence of spouse ${ }^{\mathrm{a}}$, yes/no & $311 / 91(76.8 / 22.5)$ \\
\hline Presence of child ${ }^{\mathrm{a}}$, yes/no & $344 / 38(84.9 / 9 \cdot 4)$ \\
\hline \multicolumn{2}{|l|}{ Education $^{\mathrm{a}}$} \\
\hline$\leq$ Elementary school graduate & $79(19 \cdot 5)$ \\
\hline$\leq$ Middle school-High school graduate & $195(48.1)$ \\
\hline$\geq$ University graduate & $195(48.1)$ \\
\hline Having a religion ${ }^{\mathrm{a}}$, yes/no & $184 / 211(45 \cdot 4 / 52.1)$ \\
\hline \multicolumn{2}{|l|}{ Stage } \\
\hline $1-2$ & $90(22.2)$ \\
\hline 3 & $94(23.2)$ \\
\hline 4 & $221(54.6)$ \\
\hline \multicolumn{2}{|l|}{ Chemotherapy goal } \\
\hline Primary & $27(6.7)$ \\
\hline Neoadjuvant/adjuvant & $168(41.5)$ \\
\hline Palliative & $210(51.9)$ \\
\hline \multicolumn{2}{|l|}{ Type of cancer } \\
\hline Gastrointestinal tract cancer & $138(34.1)$ \\
\hline Lung cancer & $90(22.2)$ \\
\hline Breast cancer & $87(21.5)$ \\
\hline Hepatobiliary tract cancer & $31(7 \cdot 7)$ \\
\hline Genitourinary tract cancer & $21(5.2)$ \\
\hline Head and neck cancer & $16(3.9)$ \\
\hline Gynecologic cancer & $11(2.7)$ \\
\hline Others & $11(2.7)$ \\
\hline
\end{tabular}

Values are presented as mean \pm SD or number (\%).

${ }^{\text {a}}$ Patient's number may be incorrect due to not ranked on medical chart.

${ }^{b}$ We used this term when the chemotherapy goal was not considered as neoadjuvant/adjuvant, and palliative aim. and percentages in parentheses. The comparison of patient groups between presence of surrogates' signing or not, was analyzed with the chi-square test and Student $t$ test. The factors associated with surrogate's signing, according to patient's demographics and details of chemotherapy, consent were distinguished with the use of univariate and multivariate logistical regression. All statistical tests were two-sided and interpreted at the significance level of $5 \%$. All outputs were produced using IBM SPSS Statistics 20 Professional for Medical Science version 20.0 (IBM Co., Armonk, NY, USA).

\section{RESULTS}

\section{Patients' characteristics}

Table 1 shows patient characteristics. A total of four hundred five patients, who had chemotherapy after acquisition of chemotherapy consent, were included. There were more men (60.2\%) than women and a proportion of patients had a spouse (76.8\%), had children (84.9\%), and below a high school degree (67.6\%). In addition, $54.6 \%$ of patients were stage 4 and $51.9 \%$ were provided palliative chemotherapy. The most common tumors were gastrointestinal tract cancer, followed by lung cancer (22.2\%), breast cancer (21.5\%), and others.

\section{Characteristics relating to chemotherapy consent}

Table 2 presents the characteristics of chemotherapy

Table 2. Characteristics relating to patient's chemotherapy consent $(n=405)$

\begin{tabular}{lc}
\hline Characteristic & No. (\%) \\
\hline Place of acquisition & $275(67.9)$ \\
Ward & $130(32.1)$ \\
\hline Outpatient & \\
Principal provider & $159(39.3)$ \\
Nurse specialist & $152(37.5)$ \\
Resident & $94(23.2)$ \\
\hline Professor & \\
Principal person who signed & $287(70.9)$ \\
Patient & $84(20.7)$ \\
\hline Surrogate & $34(8.4)$ \\
\hline Patient \& surrogate
\end{tabular}


Table 3. Comparison of patient's characteristics based on presence of surrogate's signing $(n=405)$

\begin{tabular}{lccc}
\hline \multirow{2}{*}{ Characteristic } & \multicolumn{2}{c}{ Surrogates' signing } & \multirow{2}{*}{ p value } \\
\cline { 2 - 3 } & No $(\mathrm{n}=321)$ & Yes $(\mathrm{n}=84)$ & \\
\hline Age & $61.0 \pm 11.43$ & $65.2 \pm 12.63$ & 0.003 \\
$\quad$ Male & $196(61.1)$ & $48(57.1)$ & 0.514 \\
\hline Female & $125(38.9)$ & $36(42.9)$ & \\
Presence of spouse & & & \\
Yes & $258(80.9)$ & $53(63.9)$ & 0.001 \\
No & $61(19.1)$ & $30(36.1)$ & \\
Presence of child & & & \\
Yes & $275(90.5)$ & $69(88.5)$ & 0.599 \\
No & $29(9.5)$ & $9(11.5)$ & \\
\hline
\end{tabular}

Education

$\begin{array}{lccc}\begin{array}{l}\text { Elementary } \\ \text { school graduate }\end{array} & 57(20.7) & 22(34.4) & 0.009 \\ \begin{array}{l}\text { Middle-High } \\ \text { school graduate }\end{array} & 158(57.5) & 37(57.8) & \\ \begin{array}{l}\geq \text { University } \\ \text { graduate }\end{array} & 60(21.8) & 5(7.8)\end{array}$

Having a religion

$\begin{array}{llll}\text { Yes } & 146(46.1) & 38(48.7) & 0.673 \\ \text { No } & 171(53.9) & 40(51.3) & \end{array}$

Chemotherapy goal

$\begin{array}{lccc}\text { Primary } & 20(6.2) & 7(8.3) & 0.000 \\ \begin{array}{l}\text { Neoadjuvant \& } \\ \text { Adjuvant }\end{array} & 150(46.7) & 18(21.4) & \\ \text { Palliative } & 151(47) & 59(70.2) & \\ \text { Stage } & & & \\ 1-2 & 81(25.3) & 9(10.7) & 0.000 \\ 3 & 82(25.6) & 12(14.3) & \\ 4 & 157(49.1) & 63(75.0) & \end{array}$

Place of acquisition

of chemotherapy

consent

$\begin{array}{llll}\text { Ward } & 207(64.5) & 68(81.0) & 0.004 \\ \text { Outpatient } & 114(35.5) & 16(19.0) & \end{array}$

Principal provider

of chemotherapy

consent

\begin{tabular}{lccc} 
Nurse specialist & $139(43.3)$ & $20(23.8)$ & 0.000 \\
Professor & $87(27.1)$ & $7(8.3)$ & \\
Resident & $95(29.6)$ & $57(67.9)$ & \\
\hline
\end{tabular}

Values are presented as mean \pm SD or number $(\%)$. consent. The place of acquisition was an inpatient ward for 275 patients $(67.9 \%)$. The principle information providers were nurse specialists (39.3\%), residents (37.5\%), and professors (23.2\%). Two hundred and eighty-seven patients (70.9\%) signed consent by themselves and 8.4\% of patients signed with together with a surrogate. Meanwhile, patient's surrogate signed for 84 patients (20.7\%).

\section{Clinical parameters associated with surrogate signing}

To determine which clinical parameters are associated with surrogate signing, we compared the patient's demographics and characteristics relating to chemotherapy consent, based on the presence of a surrogate signature. A significant correlation in the surrogate signing group was observed in patients who were older, spouse's absence, lower educational level, palliative chemotherapy, stage 4, inpatients, and those who had a resident as a principle provider of chemotherapy consent (Table 3 ).

\section{Details relating to surrogate's signing of chemo- therapy consent}

The characteristics of surrogates' signing were identified. Over half of surrogate signings were performed by patients' son or daughter (60.7\%). Next, a patient's spouse was signatory for 16 patients (19.0\%). Other people who played a role as patient's surrogate were siblings (9.5\%), daughter's-in-law (3.6\%), and son's-in-law or brother in law (1.2\%).

Two prevalent reasons for the surrogate's signature replacing the patients were due to patient incapacity, expressed as 'impossible to understand due to patient's physical and/or psychologic problems' (34.5\%), and those authorizations taken from the patients' (33.3\%). The reason of 'doing harm by providing information to patients,' included family members' requests for keeping the secret of cancer diagnosis to the patient were noted for 20 patients $(23.8 \%)$ (Table 4 ).

\section{Assessment of surrogate's signing according to patient's demographics and characteristics related to chemotherapy consent}

Age, presence of spouse, education level, chemotherapy goal, stage, place of acquisition of chemotherapy consent, and principle provider of chemotherapy consent were significant factors associated with surrogate signing in univariate logistical regression analysis. After ad- 
Table 4. The characteristics of surrogates' signing on chemotherapy consent $(n=84)$

\begin{tabular}{lc}
\hline Details & No. $(\%)$ \\
\hline Relationship with patient & $51(60.7)$ \\
\hline Son or daughter & $16(19.0)$ \\
Spouse & $8(9.5)$ \\
\hline Sibling & $3(3.6)$ \\
\hline Daughter in law & $1(1.2)$ \\
\hline Mother/Father & $1(1.2)$ \\
\hline Son in law & $1(1.2)$ \\
\hline Brother in law & $3(3.6)$ \\
\hline Unknown & $29(34.5)$ \\
Reasons & \\
\hline Impossible to understand due to patient's \\
physical and/or psychologic problems \\
\hline Taken authorization from patient & $28(33.3)$ \\
\hline Do harm by providing information to patients & $20(23.8)$ \\
\hline Others & $3(3.6)$ \\
\hline Unknown & $4(4.8)$ \\
\hline
\end{tabular}

justing for statistical and/or clinical significant factors, more surrogates signings were noted when a patient did not have spouse (OR, $0.32 ; p=0.014)$, and patient's group with elementary school education were compared to the over university graduate school education ( $\mathrm{OR}, 0.22 ; \mathrm{p}=$ $0.019)$, outpatient setting $(\mathrm{OR}, 3.05 ; p=0.037)$, and provided consent from nurse specialist or resident (professor vs. nurse specialist: $\mathrm{OR}, 0.56 ; p=0.005$; resident vs. nurse specialist: OR, $5.02 ; p=0.000$ ) were factors. While, there was a tendency with surrogate signing on stage $4 \mathrm{pa}-$ tients, yet there was no statistical significance (OR, o.33; $p=0.092)$ (Table 5).

\section{DISCUSSION}

The purpose of this study aimed to investigate our current practices related to surrogate signing of chemotherapy consent from data based reviews of institutional chemotherapy consents. Of interest, surrogate's signa-

Table 5. Univariate and multivariate analyses of surrogate signing $(n=405)$

\begin{tabular}{|c|c|c|c|c|c|c|}
\hline \multirow{2}{*}{ Variable } & \multicolumn{3}{|c|}{ Univariate analyses } & \multicolumn{3}{|c|}{ Multivariate analyses } \\
\hline & OR & $95 \% \mathrm{CI}$ & $p$ value & OR & $95 \% \mathrm{CI}$ & $p$ value \\
\hline Age & 1.03 & $1.01-1.06$ & 0.004 & 1.02 & $0.98-1.06$ & 0.379 \\
\hline Sex (male vs. female) & 0.85 & $0.52-1.38$ & 0.514 & 0.99 & $0.46-2.13$ & 0.984 \\
\hline Presence of spouse (yes vs. no) & 0.42 & $0.25-0.71$ & 0.001 & 0.32 & $0.13-0.80$ & 0.014 \\
\hline Presence of child (yes vs. no) & 0.81 & $0.37-1.79$ & 0.599 & 0.99 & $0.26-3.79$ & 0.988 \\
\hline \multicolumn{7}{|l|}{ Education } \\
\hline $\begin{array}{l}\leq \text { Mid-high school graduate vs. } \leq \text { Elementary } \\
\text { school graduate }\end{array}$ & 0.61 & $0.33-1.12$ & 0.107 & 0.69 & $0.32-1.48$ & 0.343 \\
\hline $\begin{array}{l}\leq \text { University graduate vs. } \leq \text { Elementary } \\
\text { school graduate }\end{array}$ & 0.22 & $0.08-0.61$ & 0.004 & 0.22 & $0.06-0.78$ & 0.019 \\
\hline Religion (yes/no) & 1.11 & $0.68-1.83$ & 0.673 & 0.87 & $0.44-1.75$ & 0.704 \\
\hline \multicolumn{7}{|l|}{ Chemotherapy goal } \\
\hline Neoadjuvant \& Adjuvant vs. Palliative & 0.31 & $0.17-0.55$ & 0.000 & 0.57 & $0.15^{-2.22}$ & 0.418 \\
\hline Primary vs. Palliative & 0.90 & $0.36-2.23$ & 0.813 & 2.04 & $0.4-8.85$ & 0.340 \\
\hline Stage $1,2,3$ vs. 4 & 0.32 & $0.19-0.55$ & 0.000 & 0.33 & $0.09-1.20$ & 0.092 \\
\hline \multicolumn{7}{|l|}{ Place of acquisition of chemotherapy consent } \\
\hline Outpatient vs. Ward & 0.43 & $0.24-0.77$ & 0.005 & 3.05 & $1.07-8.69$ & 0.037 \\
\hline \multicolumn{7}{|l|}{ Principal provider of chemotherapy consent } \\
\hline Professor vs. Nurse specialist & 0.56 & $0.23-1.38$ & 0.206 & 0.17 & $0.05-0.59$ & 0.005 \\
\hline Resident vs. Nurse specialist & 4.17 & $2.35-7.39$ & 0.000 & 5.02 & $2.25-11.19$ & 0.000 \\
\hline
\end{tabular}

OR, odds ratio; CI, confidence interval. 
tures were noted on a quarter of patients (20.7\%) and the relevant reasons were patient's incapacity, taken authorization from patients, and concerns for doing harm to patients by providing information.

Surrogate decision making was frequently reported in advanced cancer patients, especially in end of life decision making [11-13]. While, surrogate decision making on active anticancer treatment has been unnoticed. The patient's ability for decision making correlates inversely as their illness becomes more severe in a situation such as loss of consciousness or in a rapidly deteriorating condition. However, all patients, until an evaluation determines otherwise, should be presumed competent to make their own decisions for any non-emergent procedure [14]. As the usual chemotherapy for solid cancer patients is not an emergent practice, surrogate decision making is not the rule.

The issue of patient's capacity was the most common reason for surrogate signing in our study. Given the finding with $34.5 \%$ prevalence with patient's incapability for decision making due to their physical and/ or psychologic problems, might raise a questionable argument. The representative criteria to demonstrate patient's capacity is described as four elements [15]. First, a person must be able to communicate a clear, consistent choice. Second, the patient must understand the relevant information. Third, the patient must appreciate the situation and its consequences. Finally, the patient must be able to rationally manipulate the information. In that respect, our patients with early stage cancer, with a $10.7 \%$ proportion of surrogate signing, raised a doubt whether a physician's evaluation and assessment of a patient's decision making capacity was performed. The rational concern of doing harm, by providing information about cancer diagnosis to patients, accounts for the surrogate's signing irrespectively to patient's capacity. However, the surrogate should reconsider whether information really does harm to the patients. Legally, the decision for medical treatment of a procedure could be carried out by patient's themselves when the patient has decision making competency, unless he/she is minor. In fact, patient's decision making capacity and exploring information needs are hard to judge especially in face with serious life threatening disease and whether they are ready to listen about 'diagnosis' and decide on treatment.

Power of attorney is an instrument in writing whereby one person, the principle, appoints another as his agent and provides that agent with the authority to act on behalf of the principle [16]. We found that consent forms from four institutions demanded power of attorney if patients delegated authority to their surrogate regarding treatment decisions for chemotherapy. However, the power of attorney was not observed in all patients with surrogate signing for reasons as taken authorization from a patient in our analysis. Clearly, making the patient's capacity evaluation, documentation of power of attorney in case of authorization from a patient, and identification of patient's information need should be verified toward weighting heavily to the patient's autonomy these days.

Because there are few studies with surrogate signing on chemotherapy consent, findings of our study with significantly more surrogate signing in patient's group with absence of spouse, lower level of education, outpatient setting, and resident as a principle provider of consent, could not be directly interpreted to clinical implication. Also, we need to keep in mind that providing information for acquiring consent has both textual aspects like structure, organization, paragraphs and information elements; and contextual aspects like the situation in which the information is read [17]. This point is relevant when considering that a significant proportion of patient's signing for themselves do so because of trust in health professionals, especially if it is a professor who happens to be the principal consent provider as shown in this study.

Data from a survey of the status of medical oncology in Korea (SOMOS-K) [18], displayed that the main chemotherapy consent provider to inpatients was medical residents (57.9\%) while medical oncologist were in charge of outpatients (50.5\%). (The SOMOS-K response rate: $45.8 \%$ of medical oncologists who are full members of Korean Association of Clinical Oncology). The main provider of chemotherapy consent forms may vary based on institutional manpower or individual clinical systems. However, important issues regarding the provision of appropriate information is a prerequisite that must be kept uppermost in order for patients to make their own decisions. In fact, the question of whether surrogates really do reflect the patient's wish or preference is very ambiguous. Even more so, our study found that the priority regarding legal representatives, name- 
ly spouse, parents, and children in that order, seems not to be acknowledged, because surrogates including a patient's daughter-in-law, sibling, a son-in-law, and a brother-in-law who are not in legal priory list, played a role as a principle legal representative, even for patients who had a spouse or son/daughter present at the time consent was given.

A recent interesting study reflects a correlation between the association of 'patient's understanding goals of chemotherapy' with 'chemotherapy use' in the last month of life and hospice enrollment before death. They showed one-third of patients who recognized that chemotherapy was "not at all" likely to cure them, were no less likely than other patients to receive end of life chemotherapy, but they were significantly more likely than other patients to enroll in hospice [19]. This finding suggests our role in providing well-informed chemotherapy advice is a critical aspect of informed consent and will be important in the expected implementation in 2018 of the law for 'patient's making their own decisions toward life sustaining treatment' [20].

This study has several limitations. First, we did not attend the process of and communication between, patients and/or their surrogate and physician while chemotherapy informed consent was being acquired. In fact, there is a possibility that a surrogate signed on the consent form even the patient played a role for decision making on chemotherapy. Because we gathered information based on a retrospective review, real understanding and making informed decisions could not be interpreted by this study design. Second, the exact reason for surrogate's signing might not be demonstrated properly due to evaluation limited to medical records. In fact, there were frequently written representative reasons for surrogate signing for each institution. Because we could not collect the patient's physical and psychological competency for decision making or confirmation for authorization taken from a patient, this issue needs to be solved prospectively.

Third, this study was performed by researchers of the ethics subcommittee of palliative medicine subgroup of KCSG, other institutional data was not analyzed. This point seems to affect the factors for surrogate signing. Even the patient's group who had some characteristics such as absence of spouse, lower education level, outpatient setting, and provision for consent by resident displayed a trend on surrogate signing, these findings would not be generalized as discriminating the parameter of surrogate signing for chemotherapy consent. Despite these limitations, our study is the first report to investigate the situation of surrogate signing on chemotherapy decision making, to our knowledge.

The ethical imperative to make informed decisions for chemotherapy, based on patient values and preferences between physician and patients' themselves, is a delicate and complicated task. This covers the issue from providing objective estimations of patient survival while maintaining hope that treatment will, to relieving patient's nocebo psychologic effects of chemotherapy. We need to train ourselves as leading experts to guide cancer patients in the right direction, weighing the patient's best interest and based on the 'do no harm' principle to arrive at properly shared decisions. Further research to shed light on the ethical dilemma of chemotherapy informing practices will be needed.

\section{KEY MESSAGE}

1. Surrogate signing was noted in $20.7 \%$ of chemotherapy consent and the prevalent reasons were due to patient's physical or psychological incapacity, taken authorization from a patient, and patient protection from harmful effects by providing information.

2. Inevitable situation for surrogate signing needs to be discriminated for better enhancing patient's autonomy on acquisition for chemotherapy consent.

\section{Conflict of interest}

No potential conflict of interest relevant to this article was reported.

\section{REFERENCES}

1. Sharpe G. Consent to medical treatment. Can Med Assoc J 1977;117:692-694.

2. Schachter M, Fins JJ. Informed consent revisited: a doctrine in the service of cancer care. Oncologist 2008;13:1109-1113. 
3. Mazur DJ. What should patients be told prior to a medical procedure? Ethical and legal perspectives on medical informed consent. Am J Med 1986;81:1051-1054.

4. ASCO Institute for Quality. Informed consent for chemotherapy administration [Internet]. Alexandria (VA): American Society of Clinical Oncology, c2018 [cited 2018 Mar 8]. Available from: http://www.instituteforquality.org/ informed-consent-chemotherapy-administration.

5. Korean Academy of Medical Sciences. Chemotherapy consent form of Korean Academy of Medical Sciences [Internet]. Seoul (KR): KAMS, 2017 [cited 2018 Mar 8]. Available from: http://kams.or.kr/pds/agree/index. php?code=agree\&page=17\&keyfield=\&key=.

6. Lee HN. Patient's autonomy [Internet]. Seoul (KR): Lawtimes, 2012 [cited 2018 Mar 8]. Available from: https:// www.lawtimes.co.kr/Legal-Opinion/Legal-OpinionView?serial=65256.

7. Degner LF, Kristjanson LJ, Bowman D, et al. Information needs and decisional preferences in women with breast cancer. JAMA 1997;277:1485-1492.

8. Sarradon-Eck A, Capodano G, Bureau E, Julian-Reynier C. The surrogate: partner in the shared decision-making. Bull Cancer 2016;103:632-642.

9. Lee HR, Lim C, Yun HG, Kang SH, Kim DY. Making an informed decision of Korean cancer patients: the discrepancy between a patient's recall of information and the information needed for acquisition of radiotherapy informed consent. Support Care Cancer 2018;26:297-303.

10. Lee JK, Keam B, An AR, et al. Surrogate decision-making in Korean patients with advanced cancer: a longitudinal study. Support Care Cancer 2013;21:183-190.

11. Kim DY, Lee KE, Nam EM, et al. Do-not-resuscitate orders for terminal patients with cancer in teaching hospitals of Korea. J Palliat Med 2007;10:1153-1158.

12. Hauke D, Reiter-Theil S, Hoster E, Hiddemann W, Winkler EC. The role of relatives in decisions concerning life-prolonging treatment in patients with end-stage malignant disorders: informants, advocates or surrogate decision-makers? Ann Oncol 2011;22:2667-2674.

13. Liu TW, Wen FH, Wang CH, et al. Terminally ill Taiwanese cancer patients' and family caregivers' agreement on patterns of life-sustaining treatment preferences is poor to fair and declines over a decade: results from two independent cross-sectional studies. J Pain Symptom Manage 2017;54:35-45.

14. Glezer A, Stern TA, Mort EA, Atamian S, Abrams JL, Brendel RW. Documentation of decision-making capacity, informed consent, and health care proxies: a study of surrogate consent. Psychosomatics 2011;52:521529.

15. Appelbaum PS. Clinical practice. Assessment of patients' competence to consent to treatment. N Engl J Med 2007;357:1834-1840.

16. Wikipedia. Power of attorney [Internet]. Wikipedia, 2018 [cited 2018 Mar 8]. Available from: https://en.wikipedia. org/wiki/Power_of_attorney.

17. Sand K, Loge JH, Berger O, Gronberg BH, Kaasa S. Lung cancer patients' perceptions of informed consent documents. Patient Educ Couns 2008;73:313-317.

18. Kim DY, Lee YG, Kim BS. Survey of medical oncology status in Korea (SOMOS-K): a national survey of medical oncologists in the Korean Association for Clinical Oncology (KACO). Cancer Res Treat 2017;49:588-594.

19. Mack JW, Walling A, Dy S, et al. Patient beliefs that chemotherapy may be curative and care received at the end of life among patients with metastatic lung and colorectal cancer. Cancer 2015;121:1891-1897.

20. Korea Ministry of Government Legislation. Law for 'patient's making their own decisions toward life sustaining treatment [Internet]. Sejong (KR): Korea Ministry of Government Legislation, 1997 [cited 2018 Mar 8]. Available from: http://www.law.go.kr/lsInfoP. do?lsiSeq=180823\#0000. 